\title{
Emotional state affects gait initiation in individuals with Parkinson's disease
}

\author{
Kelly M. Naugle • Chris J. Hass • Dawn Bowers • \\ Christopher M. Janelle
}

Published online: 23 December 2011

(C) Psychonomic Society, Inc. 2011

\begin{abstract}
The purpose of the present study was to determine the impact of manipulating emotional state on gait initiation in persons with Parkinson's disease (PD) and healthy older adults. Following the presentation of pictures that are known to elicit specific emotional responses, participants initiated gait and continued to walk for several steps at their normal pace. Reaction time, the displacement and velocity of the center of pressure (COP) trajectory during the preparatory postural adjustments, and length and velocity of the first two steps were measured. Analysis of the gait initiation measures revealed that exposure to (1) threatening pictures, relative to all other pictures, speeded the initiation of gait for PD patients and healthy older adults; (2) approach-oriented emotional pictures (erotic and happy people), relative to withdrawal-oriented pictures, facilitated the anticipatory postural adjustments of gait initiation for PD patients and healthy older adults, as evidenced by greater displacement and velocity of the COP movement; and (3) emotional pictures modulated gait initiation parameters in PD patients to the same degree as in healthy older adults. Collectively, these findings hold
\end{abstract}

\section{K. M. Naugle $(\bowtie)$}

Comprehensive Center for Pain Research, University of Florida, 1329 SW 16th Street, Rm 5180, P.O. Box 103628, Gainesville, FL 32610, USA

e-mail: knaugle@dental.ufl.edu

\section{J. Hass · C. M. Janelle}

Department of Applied Physiology and Kinesiology, University of Florida,

Gainesville, FL, USA

\section{Bowers}

Department of Public Health and Health Psychology, University of Florida,

Gainesville, FL, USA significant implications for understanding the circuitry underlying the manner by which emotions modulate movement and for the development of emotion-based interventions designed to maximize improvements in gait initiation for individuals with PD.

Keywords Emotion $\cdot$ Motor $\cdot$ Basal ganglia

A growing body of literature supports the long-held notion that human emotions have evolved as fundamental action dispositions, facilitating behaviors essential for survival (Frijda, 2009; Lang, 1995). As such, emotions motivate behavioral responses to approach pleasant and avoid unpleasant stimuli and situations. Until recently, much of the data supporting this premise were incurred through the exclusive implementation of upper-extremity movements and simple measures of response speed (e.g., Chen \& Bargh, 1999; Duckworth, Bargh, Garcia, \& Chaiken, 2002). Addressing this limitation, several studies have recently evaluated the impact of emotional state on whole-body movements that clearly represent pure approach- and avoidance-related behavior (Gélat, Coudrat, \& Le Pellec, 2011; Naugle, Hass, Joyner, Coombes, \& Janelle, 2011; Naugle, Joyner, Hass, \& Janelle, 2010; Stins \& Beek, 2011). Importantly, this evidence has indicated that pleasant emotional states facilitate the initiation of approach-oriented gait in healthy individuals (Naugle et al., 2011).

Understanding emotion's impact on initiation of gait is critical for several reasons. First, measurement of movement kinematics and kinetics during the planning, initiation, and regulation of human gait initiation provides an ideal opportunity to evaluate how various components of the motor system are altered by emotion. Second, gait initiation is an activity of 
daily living task that is challenging to the motor control system because it is a volitional transition from a condition of a static stable support to a continuously unstable posture during locomotion. Not surprisingly, then, impaired gait initiation performance is common in older adults and those with disability. Third, given prior findings, manipulating emotional state may be a potentially efficacious strategy for enhancing gait initiation parameters in persons with movement disorders, such as Parkinson's disease (PD). The primary aim of this study was to determine whether and how emotional manipulations would alter gait initiation in older adults with and without PD. Fulfillment of this aim permitted inferences to be made concerning the integrity of parallel and integrated emotion and motor circuits in persons with and without known deficits in basal ganglia function, as compared with their age-matched counterparts, and as compared with the kinetic metrics of gait initiation identified in previous work with young adults (e.g., Naugle et al., 2011).

\section{Emotion and gait initiation}

Kinematic and kinetic analyses of gait initiation can be used to determine how various components of the motor system are altered by emotion. Gait initiation is considered the phase between motionless standing and rhythmic walking, requiring effective balance control as forward momentum is generated (Halliday, Gai, Blessing, \& Geffen, 1990). Gait initiation includes preparatory and stepping components, both of which are regulated by partially different circuits in the brain (Rocchi, Chiari, Cappello, \& Horak, 2006; Takakusaki, Habaguchi, Ohtinata-Sugimoto, Saitoh, \& Sakamoto, 2003). During the preparatory phase and prior to the initiation of the stepping movement, anticipatory postural adjustments (APAs) uncouple movement of the center of mass (COM) and the center of pressure (COP). Specifically, these postural adjustments shift the COP backward and laterally toward the initial swing (stepping) limb to move the COM forward and toward the stance limb (APA phase; Crenna, Frigo, Giovannini, \& Piccolo, 1990). This backward shift initiates the forward momentum needed to take a step. The lateral shift of the COP toward the swing limb propels the COM toward the stance (support) limb, in preparation for single limb support, and preserves lateral stability during step execution (Jian, Winter, Ishac, \& Gilchrist, 1993; Polcyn, Lipsitz, Kerrigan, \& Collins, 1998; Zettel, McIlroy, \& Maki, 2002). Thereafter, the COP moves rapidly toward the stance limb as the swing limb unloads in preparation for foot-off (Crenna et al., 1990; Jian et al., 1993). Finally, the COP moves anteriorly until toe-off of the initial stance limb (locomotor phase). The stepping components of gait initiation include the execution of the first and second steps.
Critical to the present proposal, we recently showed that emotional state differentially influences the preparatory and stepping components of forward gait initiation in healthy young adults (Naugle et al., 2011). Viewing highly arousing unpleasant stimuli (i.e., attack images) speeded the initial motor response on an approach-oriented gait initiation task, as compared with all other affective stimuli. This finding supported prior research showing that threatening cues prime the motor system for action regardless of movement direction (i.e., approach vs. avoidance; Coombes, Cauraugh, \& Janelle, 2007; Coombes, Higgins, Gamble, Cauraugh, \& Janelle, 2009). As the direction of the movement emerged during the gait initiation task, the presentation of the pleasant stimuli facilitated the initiation of forward gait as indexed by greater posterior and lateral COP movement toward the swing limb during the APAs, as well as greater velocity of the COP shift. Furthermore, the first step was executed with increased velocity following the presentation of pleasant images, as compared with unpleasant images, while no impact of emotion was observed on the second step. These data provided evidence that emotional state systematically alters gait initiation, with a greater impact on the planning and preparatory components than on the more rhythmic locomotor components.

\section{Posture and gait performance in PD}

PD is a progressive neurodegenerative disease of the basal ganglia characterized by debilitating motor symptoms and emotional dysfunction. One of the most debilitating motor symptoms of PD is postural instability and gait dysfunction. Despite the benefits of current pharmacological and surgical therapies for patients with PD, treatment effectiveness and options for postural instability and gait difficulties remain limited (Rodriguez-Oroz et al., 2005; Sethi, 2008).

In PD, the dopaminergic denervation of the striatum causes abnormalities in the brain circuits regulating gait initiation, resulting in inefficient APAs and increased movement preparation time (Burleigh-Jacobs, Horak, Nutt, \& Obeso, 1997; Crenna et al., 1990; Halliday, Gai, Blessing, \& Geffen, 1998). In particular, the initial posterior and lateral COP movement toward the swing foot during the APAs is reduced and slower in persons with PD. Additionally, the initial steps of persons with PD are characterized by reduced step length and velocity, as compared with age-matched controls (Crenna et al., 1990). Collectively, research has shown that gait initiation parameters in persons with PD (relative to healthy controls) are smaller, slower, and less forceful. As the disease progresses, these gait abnormalities become more pronounced, limiting quality of life (Morris, Iansek, Smithson, \& Huxham, 2000). Although both the postural and stepping components are improved by anti-Parkinsonian 
medication (Sethi, 2008), gait initiation performance remains impaired, relative to age-matched controls, even when persons with PD are optimally medicated.

\section{Emotion deficits in PD}

The nigrostriatal neuronal degeneration in PD causes dysfunction not only in motor circuits, but also in limbic pathways, such as the mesocorticolimbic dopaminergic pathway. Additionally, individuals with PD exhibit pathological changes in structures of limbic circuits, including the amygdala and ventral tegmental area (Harding, Stimson, Henderson, \& Halliday, 2002; Uhl, Hedreen, \& Price, 1985). Not surprisingly, then, PD is increasingly linked with emotional dysfunction, such as deficits in perception of emotional expression and greater symptoms of depression, anxiety, and apathy (McDonald, Richard, \& DeLong, 2003; Slaughter, Slaughter, Nichols, Holmes, \& Martens, 2001). Research has also revealed that, as compared with healthy controls, individuals with PD exhibit reduced psychophysiological reactivity (i.e., blunted startle eye blink magnitude) to highly arousing disgust-oriented pictures, but similar reactivity in response to attack, contamination, pleasant, and neutral pictures (Bowers et al., 2006). While empirical investigations have examined how emotion alters involuntary movement (i.e., startle eye blink) in PD, researchers have yet to explore whether persons with PD show abnormalities in emotional reactivity as indexed by voluntary whole-body movements.

\section{The present study}

The purpose of the proposed project was to determine the impact of emotional state on the quality of gait initiation in persons with PD while on their normal dopaminergic medication. Animal research suggests that emotion is integrated into motor circuits via midbrain dopamine neurons in a striato-nigro-striatal pathway (Haber, 2003; Haber \& Calzavara, 2009). Critically, individuals with PD exhibit atypical activation within motor and limbic basal ganglia circuits via loss of dopaminergic neurons in the substantia nigra (SN), causing motor and emotional dysfunction. However, during "on" states when standard anti-Parkinsonian pharmacological treatments normalize dopamine levels, we predict that emotion information will be successfully integrated into the motor system, allowing emotional input to impact the quality of intended movements. If we demonstrate that emotional reactivity of $\mathrm{PD}$ patients is similar to that of age-matched controls, we will know that the integrity of the underlying circuitry remains intact (or facilitated by medication) in PD patients.
Alternatively, if we show that PD patients differ from agematched controls, such findings will indicate that the circuitry that underlies emotion-modulated movement in the neurologically healthy is compromised in PD patients.

In the present study, individuals with PD and healthy aged-matched controls initiated gait and continued to walk following exposure to attack, mutilation, contamination, erotica, happy people, neutral, and blank pictures. Of particular interest was to determine the extent to which specific emotion categories would alter the speed of movement initiation (reaction time [RT]), the quality of the postural adjustments during gait initiation (as indexed by COP displacements and velocities), and step execution (as evidenced by the length and velocity of the first and second steps). We hypothesized that for all participants, (1) exposure to attack pictures would lead to significantly shorter RTs on the gait initiation task, as compared with all other picture categories, and (2) exposure to the approachrelated categories of erotica and happy people, as compared with the withdrawal-related categories (mutilation and contamination), would facilitate forward gait initiation, as evidenced by greater displacement and velocity of the posterior and lateral COP movement during the APA phase and by greater length and velocity of the first step.

\section{Method}

\section{Participants}

Participants included 26 patients with idiopathic PD and 26 age-matched controls. Patients with PD were recruited through the university's Center for Movement Disorders and Neurorestoration and local neurology offices and from physical and occupational therapy practices in the community. The control participants were recruited from the same community and were age and gender matched to the PD patients. All participants with PD were tested in their best medicated state, approximately $1-1.5 \mathrm{~h}$ following a dose of anti-Parkinsonian medication. All participants were fully informed of the nature of the study and their right to decline participation or withdraw from participation at any point of time. Written informed consent for participation was obtained according to university and federal guidelines. See Table 1 for the inclusion and exclusion criteria for participants.

Instrumentation

Emotion manipulation Picture viewing was used to induce emotional states during experimental trials. Presented stimuli included 30 digitized photographs selected from the International Affective Picture System (IAPS: Lang, Bradley, \& 
Table 1 Participant inclusion and exclusion criteria

Inclusion criteria for persons with PD

1. Clinical diagnosis of idiopathic PD (Hughes, Daniel, Kilford, \& Lees, 1992) - presence of at least 2 or 3 cardinal signs of PD

2. Complaints of persistent gait disturbance despite optimal medical therapy-score $\geq 1$ on item 29 on motor portion of UPDRS

3. Modified Hoehn and Yahr stage between 2.0 and 4.0 in the "off" state

4. Age of $55-80$ years

5. Stable regimen of anti-Parkinsonian and psychotropic drug therapy for 30 days prior to study

Exclusion criteria for persons with PD

1. Atypical Parkinsonian features

2. Medications affecting balance or alertness/attention

3. "On" freezers - score $\geq 1$ on item 14 on the Activities of Daily Living subsection of UPDRS

Exclusion criteria for all participants

1. Neurological disturbance (other than PD for PD group) or chronic medical illness

2. History of major psychiatric disorder

3. Peripheral neuropathy, orthopedic, vestibular, assisted devices

4. History of head injury, epilepsy, stroke, or learning disability

5. Dementia - score $<26 / 30$ on Montreal Cognitive Assessment (MOCA: Nasreddine et al., 2005)

6. Lower extremity injuries that would affect movement

7. Severe symptoms of depression - score $<19$ on Beck Depression Inventory II (BDI-2: Beck, Steer, \& Brown, 1996)

8. High trait anxiety-score $<45$ on trait scale of State Trait Anxiety Inventory (STAI: Speilberger, 1983)

Cuthbert, 2008) representing six affective categories: (1) erotica, (2) happy people, (3) mutilation, (4) contamination, (5) attack, and (6) neutral. ${ }^{1}$ All pictures were chosen according to affective norms (Lang, Bradley, \& Cuthbert, 2008). Threat (i.e., pointed guns, knife attacks), mutilation (i.e., mutilated bodies and faces), and erotica (i.e., erotic couples) stimuli are rated high in arousal and strongly activate defensive and appetitive systems (Bradley, Codispoti, Sabatinelli, \& Lang, 2001), respectively. Erotic and threat pictures were included because they have been previously shown to alter gait parameters in healthy individuals (Naugle et al., 2011). While the specific effect of mutilation images on gait in healthy individuals has not been evaluated, this category was included because of research showing mutilation-specific hyporeactivity in PD (Miller, Okun, Marsiske, Fennell, \& Bowers, 2009). The happy people category (i.e., babies, children, happy couples) was included because, similar to the more arousing erotic category, it has been shown to facilitate gait initiation in healthy individuals (Naugle et al., 2011). The contamination category (i.e., dirty toilets, bugs on food) provides an unpleasant category that matches the arousal level of the

\footnotetext{
${ }^{1}$ IAPS Pictures and average normative arousal and valence ratings: attack (arousal $=6.64 \pm 2.19$; valence $=2.68 \pm 1.65$ ): 6210, 6250, $6260,6370,6510$; mutilation (arousal $=6.80 \pm 2.13$; valence $=1.82 \pm$ 1.37): 3060, 3071, 3100, 3150, 3130; contamination (arousal $=5.49 \pm$ 2.44; valence $=2.51 \pm 1.67): 9300,7359,9301,7380,9320$; erotica (arousal $=6.41 \pm 2.09 ;$ valence $=6.92 \pm 1.72): 4607,4670,4694$, 4608, 4676; pleasant $($ arousal $=5.30 \pm 2.38$; valence $=7.33 \pm 1.77$ ): $4598,2071,4623,2345,2058$; neutral (arousal $=3.18 \pm 2.01$; valence $=$ $4.77 \pm 1.30): 2190,2200,2210,2305,2104$.
}

happy people category and induces clear avoidance motivation. The neutral pictures (i.e., neutral faces) are less arousing than all affective categories. Five catch trials were also included, in which no image (a blank black screen) was presented.

Pictures were projected onto a $3.3 \times 2 \mathrm{~m}$ screen using a NEC VT 670 digital projector. The screen was located $6 \mathrm{~m}$ in front of participants. Pictures were $127 \times 91 \mathrm{~cm}$ and $1,024 \times 768$ pixels. Three randomized picture orders were created and counterbalanced across participants. The picture categories were completely randomized within each picture order. A custom LabVIEW program (LabVIEW 8.1; National Instruments, Austin, TX) was used to control trial onset, trial offset, and visual stimulus presentation. A computerized 9-point version of the self-assessment manikin (SAM: Lang, 1980) was used to obtain subjective ratings of valence and arousal at the conclusion of gait testing.

Task Participants were fitted with retro-reflective markers, which were placed bilaterally on the lower body at the following locations: anterior superior iliac, posterior superior iliac, lateral epicondyle of the knee, lower lateral one third of the surface of the thigh, lateral malleolus, tibia, second metatarsal head, and calcaneus. Once the reflective markers were in place, each participant was given the opportunity to walk around the testing environment to become accustomed to the instrumentation.

During the gait initiation trials, participants stood with their feet in a self-selected stance width, with both feet on one force platform (Bertec, Columbus, Ohio, model 4060). The positioning of the feet was recorded to allow for 
standardization for all future trials. At picture offset, participants began walking and continued for several steps (approximately $4 \mathrm{~m}$ ).

The kinematic characteristics of the locomotor tasks were sampled at a rate of $120 \mathrm{~Hz}$ using a ten-camera Optical Motion Capture system (Vicon Peak, Oxford, U.K.). The motion capture system collected three-dimensional coordinate data from retro-reflective markers. Ground reaction forces (GRFs) and COP measurements were collected at $1200 \mathrm{~Hz}$ using three Bertec force platforms (Bertec, Newton, MA; size, $60 \times 40 \mathrm{~cm}$ ) mounted flush with the laboratory floor.

\section{Procedure}

Upon arrival at the laboratory, participants completed a battery of self-report questionnaires including demographics, the state and trait forms of the STAI (Spielberger, 1983), the state version of the Positive and Negative Affect Schedule (PANAS: Watson, Clarke, \& Tellegen, 1988), the BDI-2 (Beck et al., 1996), and the Apathy Scale (Starkstein et al., 1995). The experimenter then administered the MOCA.

Following completion of questionnaires, participants were fitted with retro-reflective markers and familiarized with the protocol, completing 3 practice trials using unique neutral pictures and one blank picture. The practice trials were immediately followed by 35 data collection trials. Participants were informed that each trial would begin with the presentation of a fixation cross on the video screen (2 s), which would be replaced by a picture for 2-4 s. Participants were instructed to look at the picture the entire time it was on the screen. At picture offset, the screen became blank (white). Participants were instructed to initiate walking with their preferred limb as soon as possible following picture offset and to continue walking at their self-selected pace. Each participant performed 5 trials for each affective category and 5 catch trials using the blank black screen, for a total of 35 trials. To determine whether participants became physically fatigued during the experimental session, they also completed 5 self-initiated trials with no picture presentation before (pretrials) and after (posttrials) the experimental trials. Following completion of the gait initiation trials, participants completed the computerized SAM scale to provide an arousal and valence rating (scale: 1-9) for each picture previously viewed.

\section{Data reduction}

RT, displacement and velocity of COP, step length, and average step velocity of the first and second steps were calculated. Trials on which participants did not initiate gait following picture offset (i.e., did not initiate gait at all or initiated gait prior to picture offset) were removed. No more than one trial for each category condition was removed for each participant.

Reaction time RT was operationalized as the latency from the movement trigger (picture offset) to the initiation of the motor response. Initiation of motor response was defined as the time at which the differentiation in the GRFs from quiet stance reaches a $5 \%$ threshold of force production (Diermayr, Gynsin, Hass, \& Gordon, 2008).

COP displacement and velocity Movement of the COP trajectory was quantified by the displacements and velocities of the COP trace observed over time in both the medio-lateral (MP) and anterior-posterior (AP) directions. The COP trace during the gait initiation trials was divided into three periods (S1, APA; S2, weight transfer; S3, locomotor) by identifying two landmark events (Hass et al., 2004) (see Fig. 1).

During these three periods, the following dependent variables were evaluated: (1) average displacement of the COP in the AP and ML directions and (2) average velocity of the $\mathrm{COP}$ in the $\mathrm{AP}$ and $\mathrm{ML}$ directions.

Step length and velocity Step length of the first step was calculated as the displacement (in centimeters) of the initial swing limb heel marker from its initial resting position until heel strike. Step length of the second step was calculated as the displacement (in centimeters) from the heel position of the swing leg at first heel strike to the heel position of the

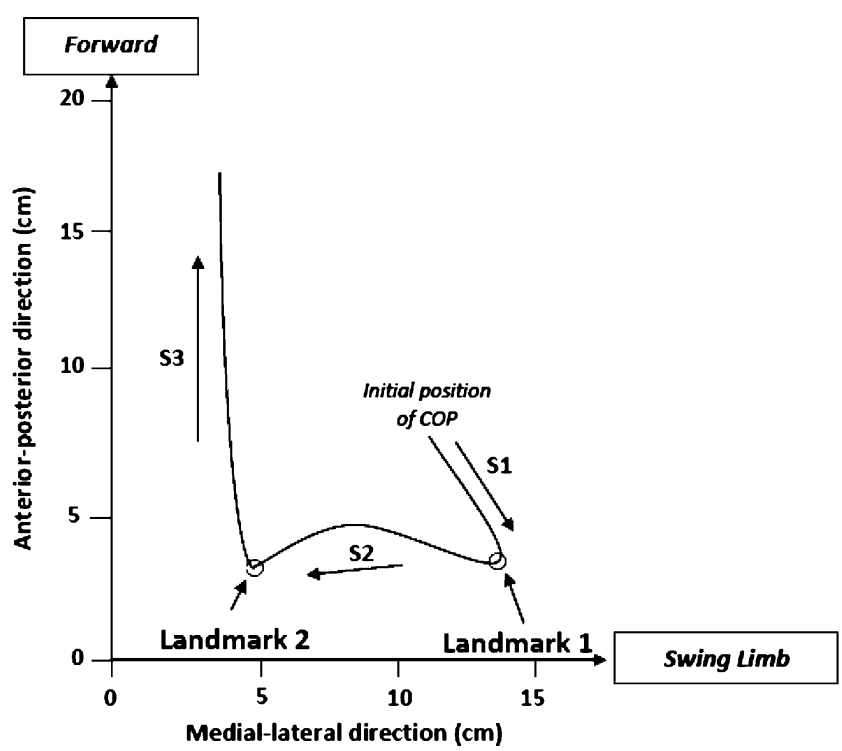

Fig. 1 S1 begins with picture offset and ends with the center of pressure (COP) located in its most posterior and lateral position toward the initial swing limb (landmark 1). S2 is defined as the translation of the COP toward the stance limb ending at landmark 2 , which is the position under the stance limb on which the COP begins to move forward under the foot. $\mathrm{S} 3$ begins at landmark 2 until the frame prior to heel strike of the swing limb as the COP is translated anteriorly 
stance leg at heel strike. An average velocity of each step was calculated as the step length divided by the corresponding change in time (in centimeters per second).

Percent change scores Replicating previous research (Naugle et al., 2011), we created a single index for each movement variable that represented the change in movement due to each affective category, relative to the neutral category. Because PD patients exhibited smaller and slower COP adjustments and steps than did the control participants, percent change scores, rather than raw bias scores, were used to remove the influence of baseline differences. Percent change scores were calculated with the following formula: [(emotional category/ neutral category) $* 100]-100$. A positive score therefore indicates greater values for the dependent variable during the emotional category, relative to the neutral category, while a negative score indicates reduced values for the dependent variable during the emotional category, relative to the neutral category. Percent change scores were the basis for the primary statistical analyses.

\section{Statistical analyses and results}

\section{Participants}

Twenty-six individuals with idiopathic PD patients (female $=$ 3 ) and 25 aged-matched ( \pm 3 years) controls (females $=3$ ) participated in this study. Three male PD patients were excluded from statistical analyses: Two patients scored above 19 on the BDI, and 1 patient scored below 26 on the MOCA. After the removal of these participants, the PD group included 23 individuals. Descriptive characteristics were calculated for each group for age, height, weight, and all affective state and trait measures (see Table 2). Independent samples $t$-tests were used to determine whether the PD and control groups differed on any of the descriptive characteristics. As is shown in Table 2, the PD and control groups did not differ statistically with respect to age, mass, cognitive dysfunction, trait anxiety, and positive and negative affect. PD patients obtained significantly higher scores than did controls on the BDI-2, Apathy Scale, and state version of the STAI. However, both groups had mean BDI scores in the nondepressed range and mean STAI-S scores indicating a low to moderate level of state anxiety. The PD patients were in the moderate stages of their disease, on the basis of Hoehn and Yahr staging and UPDRS motor scores (Fahn, Elton, \& UPDRS Development Committee, 1987).

\section{Performance on pre- versus posttrials}

To determine whether fatigue may have influenced the gait initiation trials as the experimental session progressed, the raw COP and step variables for the pre- and posttrials were each analyzed with 2 (group: PD, control) $\times 2$ (time: pre, post) MANOVAs with repeated measures on the second factor. These analyses revealed that performance did not decline on the gait initiation task from the pretrials to the posttrials, indicating that fatigue was likely not a factor during the experimental trials $(p s>.05)$.

\section{Effect of affective trait variables and PD on performance}

The raw values for each dependent variable were analyzed with hierarchical linear modeling (HLM) to determine whether depression, apathy, and the presence of PD (1) affected the gait initiation variables after controlling for height and (2) moderated the relationship between emotion (self-reported valence and arousal ratings) and the gait initiation variables. The results showed that level of
Table 2 Demographic, affective, and clinical characteristics by group

Note. UPDRS Motor: motor scale of the Unified Parkinson Disease Rating Scale.

*Significant

\begin{tabular}{lccc}
\hline Characteristic & $\begin{array}{l}\text { PD }(n=23) \\
\text { Mean }(S D)\end{array}$ & $\begin{array}{l}\text { Control }(n=25) \\
\text { Mean }(S D)\end{array}$ & Statistical Test \\
\hline Age & $70.13(7.63)$ & $67.20(4.97)$ & $t(46)=1.56, p=.13$ \\
Mass (kg) & $76.92(9.46)$ & $80.65(14.91)$ & $t(46)=-1.03, p=.31$ \\
Height (cm) & $168.09(4.72)$ & $172.21(6.86)$ & $t(46)=-2.41, p=.02^{*}$ \\
Trait anxiety (STAI-T) & $32.13(7.70)$ & $29.40(6.14)$ & $t(46)=1.36, p=.18$ \\
Depression (BDI-2) & $007.91(4.86)$ & $4.72(3.05)$ & $t(46)=2.70, p=.01^{*}$ \\
Apathy (Apathy Scale) & $13.17(6.58)$ & $8.16(3.00)$ & $t(46)=3.35, p<.01^{*}$ \\
State anxiety (STAI-S) & $32.22(9.42)$ & $26.64(6.61)$ & $t(46)=2.36, p=.02^{*}$ \\
PANAS Positive scale & $33.91(7.37)$ & $37.48(5.06)$ & $t(46)=-1.96, p=.06$ \\
PANAS Negative scale & $12.95(3.93)$ & $11.16(2.38)$ & $t(46)=1.91, p=.06$ \\
Cognitive dysfunction & & & $t(46)=0.52, p=.96$ \\
(MOCA) & $27.74(1.45)$ & $27.72(1.02)$ & \\
Hoehn and Yahr stage & $2.95(0.50)$ & & \\
UPDRS Motor & $26.74(6.18)$ & & \\
\hline
\end{tabular}


depression and apathy did not impact performance on the gait initiation trials or the relationship between emotion and performance. As was expected, as compared with control participants, PD participants exhibited reduced (1) posterior COP displacement and velocity during S1, (2) medial COP velocity during S2, and (3) length and velocity of step 1.

\section{Reaction time}

RT percent change scores were analyzed with a 2 (group: $\mathrm{PD}$, control $) \times 5$ (category: erotica, happy people, mutilation, contamination, attack) ANOVA with repeated measures on the second factor. A significant main effect of category was found, $F(2.84,113.76)=6.43, p=.001, \eta^{2}=.14$. Tukey's HSD procedure demonstrated that RT was shorter following the presentation of the attack pictures, as compared with all other affective picture categories $(p \mathrm{~s}<.05)$ (see Fig. 2). The main effect of group $(p=.657)$ and the group $\times$ category interaction $(p=.881)$ were not significant.

\section{COP trace}

COP displacement and velocity percent change scores were evaluated during the three periods of the COP trace (S1, S2, S3). Thus, three separate 2 (group: PD, control) $\times 5$ (category: erotica, happy people, mutilation, contamination, attack) MANOVAs with repeated measures on the second factor were conducted. The dependent variables for each MANOVA included AP and ML displacement and AP and ML velocity. For each MANOVA, separate two-way ANOVAs were performed for follow-up testing when appropriate. Follow-up analyses were conducted using Tukey HSD procedure and simple effects tests for significant main effects and interactions, respectively.

S1 region of the COP trace The analysis revealed a significant main effect of category (Wilks's lambda $=.823$ ), $F$ $(16,480.00)=1.98, p=.013, \eta^{2}=.05$. Figure 3 illustrates the

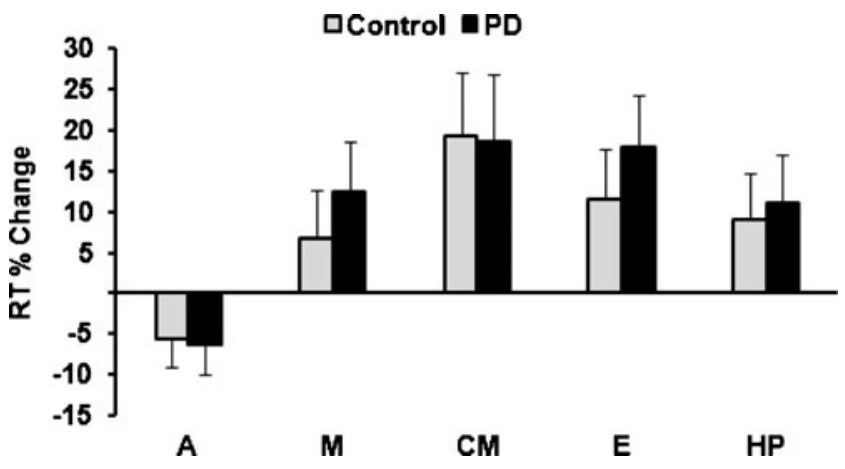

Fig. 2 Mean percent change scores for reaction time (RT) across category conditions for the Parkinson's disease (PD) and control groups. A = attack, $\mathrm{M}=$ mutilation, $\mathrm{CM}=$ contamination, $\mathrm{E}=$ erotic, $\mathrm{HP}=$ happy people percent change scores for displacement and velocity of the COP movement in the posterior and lateral directions. The respective follow-up ANOVAs showed a significant effect of category for the displacement of the COP movement in the posterior direction, $F(2.84,113.60)=5.73, p=.001, \eta^{2}=.13$. The posterior COP displacement percent change scores for the happy people and erotic pictures were significantly greater than the percent change scores for the attack and contamination pictures $(p \mathrm{~s}<.05)$ (Fig. 3a). The ANOVA conducted on the velocity of the posterior COP approached significance, $F$ $(2.56,102.49)=2.66, p=.06, \eta^{2}=.06$, with the erotic and happy people pictures trending toward greater posterior velocity percent change scores, as compared with the attack and contamination pictures. The ANOVAs also showed a significant effect of category for the COP displacement in the lateral direction, $F(4,160)=3.11, p=.017, \eta^{2}=.07$. The percent change scores for the erotic pictures were significantly greater than for the mutilation pictures, and the happy people pictures were significantly greater than the mutilation and contamination pictures $(p s<.05)$ (Fig. 3c). The ANOVA conducted on the lateral COP velocity percent change scores was not significant $(p=.182)$. The main effect of group $(p=.206)$ and the group $\times$ category interaction $(p=.178)$ were not significant.

$S 2$ region of the COP trace The main effect of category approached significance (Wilks's lambda $=.853), F(16.00$, $480.28)=1.60, p=.065, \eta^{2}=.039$, and was driven by a significant ANOVA for the velocity of the COP trajectory in the ML direction, $F(4,160)=3.35, p=.011, \eta^{2}=.052$. The main effect of group $(p=.710)$ and the group $\times$ category interaction $(p=.637)$ were not significant.

S3 region of the COP trace No significant effects were found for the percent change scores during the S3 portion of the COP trajectory $(p s>.05)$.

Average step length and step velocity of the first and second steps

Step length and velocity of the first and second steps and stride length percent change scores were analyzed in a 2 (group: PD, control) $\times 5$ (category: erotica, happy people, mutilation, contamination, attack) MANOVA with repeated measures on the second factor. The analysis produced a significant category $\times$ group interaction (Wilks's lambda $=$ $.825), F(20,544.88)=1.62, p=.043, \eta^{2}=.047$. The follow-up tests revealed a significant interaction for the velocity of the first step, $F(4,168)=3.49, p=.009, \eta^{2}=$ .08. Exposure to mutilation pictures for the PD group resulted in reduced step velocity percent changes scores, as compared with the control group's response to mutilation and erotic pictures, as well as happy people pictures for 

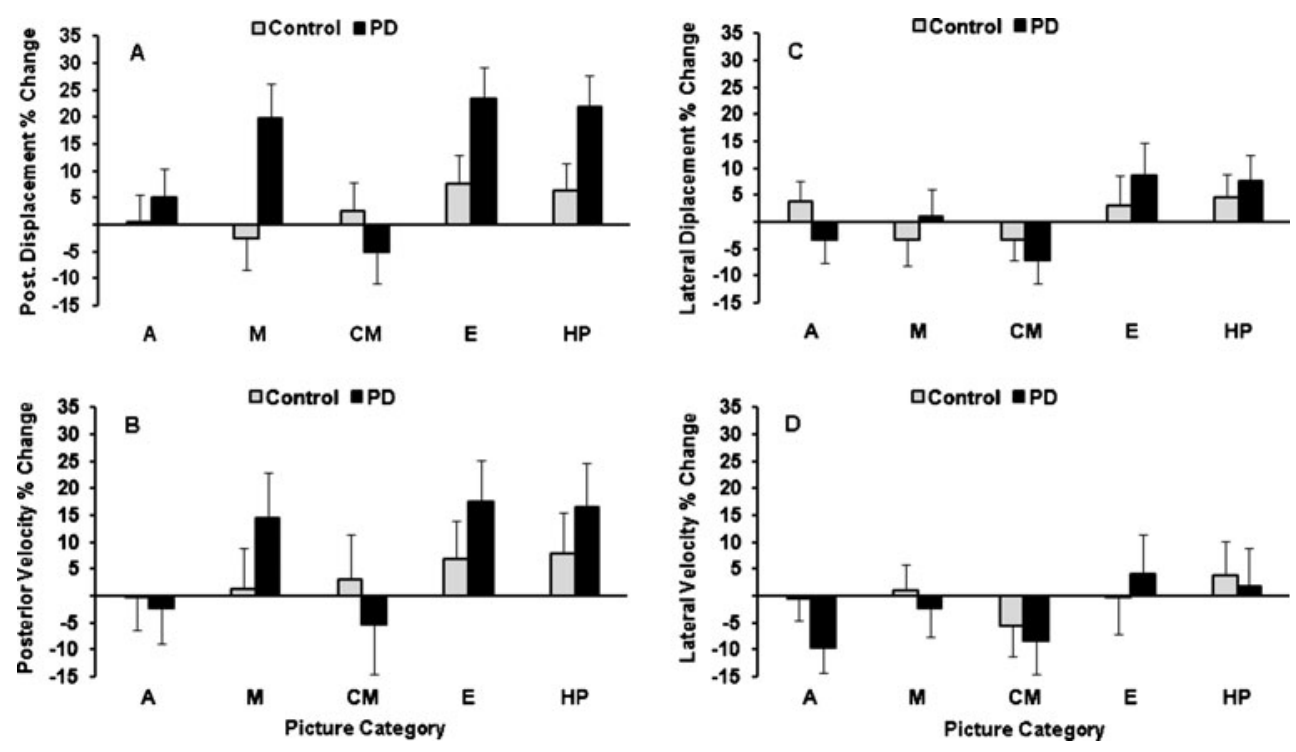

Fig. 3 Center of pressure movement percent change scores in the S1 region across category conditions for the Parkinson's disease (PD) and control groups for the mean percent change displacement in the posterior direction (a), mean percent change velocity in the posterior

direction (b), mean percent change displacement in the lateral direction (c), and mean percent change velocity in the lateral direction (d). Post. = posterior, $\mathrm{A}=$ attack, $\mathrm{M}=$ mutilation, $\mathrm{CM}=$ contamination, $\mathrm{E}=$ erotic, $\mathrm{HP}=$ happy people

both groups $(p \mathrm{~s}<.05)$ (see Fig. 4). The group $(p=.682)$ and category $(p=.686)$ main effects were not significant. Additionally, no significant findings were found for step 2.

\section{SAM ratings}

Two-way ANOVAs (group $\times$ category) were conducted on the SAM valence and arousal ratings. A significant main effect of category, $F(2.97,121.70)=305.85, p>.001, \eta^{2}=.882$, was found for the valence ratings. As was expected, all participants rated the erotic and happy people pictures as significantly more pleasant than the mutilation, attack, contamination, and neutral pictures $(p s>.05)$ (Fig. 5a). The mutilation pictures were rated significantly more unpleasant than all other picture categories, and the attack and contamination pictures were rated as more unpleasant than the neutral pictures $(p s>.05)$.

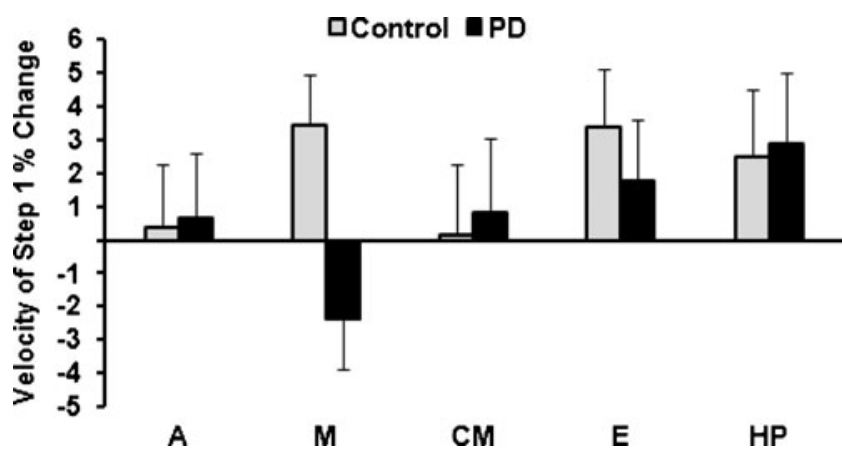

Fig. 4 Mean percent change scores for velocity of the first step across category conditions for the Parkinson's disease (PD) and control groups. $\mathrm{A}=$ attack, $\mathrm{M}=$ mutilation, $\mathrm{CM}=$ contamination, $\mathrm{E}=$ erotic, $\mathrm{HP}=$ happy people
The main effect of group $(p=.146)$ and the interaction $(p=.219)$ were not significant.

The two-way ANOVA conducted on the arousal ratings also demonstrated a significant main effect of category, $F(2.96,121.29)=41.81, p>.001, \eta^{2}=.51$. As is demonstrated in Fig. 5b, the erotic pictures were rated significantly more arousing than all other picture categories, and mutilation pictures were rated more arousing than all categories except for erotic $(p s>.05)$. Attack pictures were rated significantly more arousing than the happy people, contamination, and neutral pictures $(p s>.05)$. Finally, the contamination and happy people pictures were rated significantly more arousing than neutral pictures $(p \mathrm{~s}>.05)$. The main effect of group $(p=.090)$ and the interaction $(p=.168)$ were not significant.

\section{Discussion}

Three novel findings emerged from this study: (1) Threatening stimuli speed the initiation of the motor response, (2) pleasant emotional stimuli facilitate the APAs of gait initiation, and (3) exposure to emotional stimuli modulates gait initiation parameters in PD patients to the same degree as in healthy older adults. These findings provide important evidence concerning how the motor system operates under different emotional conditions in both PD patients while on anti-Parkinsonian medication and those with intact neurological systems. Additionally, the data suggest that manipulation of emotional state may be a viable strategy for enhancing the quality of gait initiation in individuals suffering from PD. 


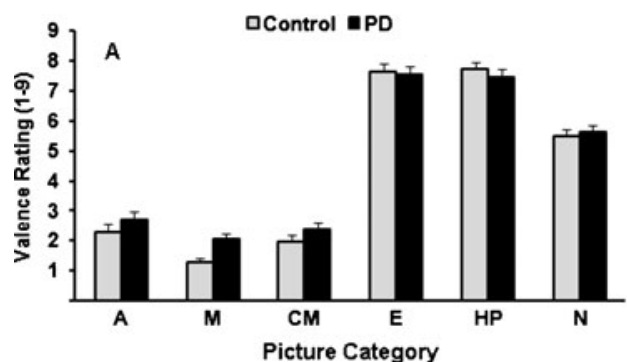

Fig. 5 Mean SAM valence ratings (a)and arousal ratings (b)across category conditions for the Parkinson's disease (PD) and control groups. The higher a participant's rating, the more the participant

Emotion's impact on RT in the gait initiation task

Consistent with our hypothesis, exposure to attack pictures speeded the motor response to all other pictures categories. The present data support and extend previous evidence showing that exposure to threatening stimuli speeds the initiation of the motor response on a gait initiation task in healthy young adults (Naugle et al., 2011), as well as on upper-extremity movement tasks (e.g., ballistic precision pinch grip, Coombes et al., 2009; wrist extension, Coombes et al., 2007). Threatening contexts theoretically prime the motor system for action, accelerating motor responses and, thereby, providing organisms the advantage of an efficient fight or flight response (Öhman, Hamm, \& Hugdahl, 2000; Öhman \& Soares, 1998). Furthermore, Coombes et al. (2007) showed that activating defensive emotional circuitry speeds the initiation of the appropriate motor action by facilitating the central processes that precede overt movement. Recent work also suggests that the neuronal circuitry mediating accelerated initiation of the APAs remains intact in PD patients, even when "off" medication (Rogers et al., 2011). Evidence from the presenst project corroborates these positions and, importantly, suggests that the neural circuitry underlying the survival function of the primitive emotion system remains preserved in healthy older adults and those with PD while "on" medication. Indeed, a similar reduction in RT $(\sim 6 \%)$ was observed for PD and control participants in response to attack pictures, relative to neutral pictures.

It is worth mentioning that our results are in contrast to those in prior studies on healthy young adults that have identified longer RTs on approach-oriented gait initiation tasks when they were confronted with unpleasant pictures, as compared with pleasant pictures (Gelat et al., 2011; Stins \& Beek, 2011). Methodological differences regarding timing of picture presentation (e.g., instructions that specified initiating gait at picture onset and walking during picture presentation vs. walking at picture offset, as was the case herein) and the categorization of unpleasant pictures may account for the contrasting responses to unpleasant pictures in the aforementioned studies. For example, studies finding longer RTs

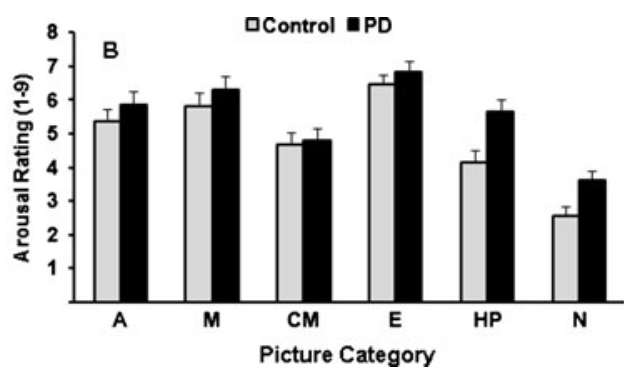

perceived the pictures as being pleasant or arousing, respectively. $\mathrm{A}=$ attack, $\mathrm{M}=$ mutilation, $\mathrm{CM}=$ contamination, $\mathrm{E}=$ erotic, $\mathrm{HP}=$ happy people, $\mathrm{N}=$ neutral

combined attack, mutilation, and contamination pictures into one unpleasant category (Gelat et al., 2011; Stins \& Beek, 2011), whereas studies finding accelerated motor responses to unpleasant pictures have looked specifically at attack pictures (Coombes et al., 2007; Coombes et al., 2009; Naugle et al., 2011). Prior work has shown that not all aversive stimuli facilitate avoidance-related behavior (Carver \& Harmon-Jones, 2009; Hess, Blairy, \& Kleck, 2000; Marsh, Ambady, \& Kleck, 2005), suggesting that behavioral responses are often emotion specific, rather than reflecting broad valence dimensions. In support of this notion, our results showed that attack pictures speeded RT, whereas mutilation and contamination pictures did not.

\section{Emotion's impact on gait initiation performance}

When PD patients and healthy older adults approached an emotional stimulus, the approach-oriented (i.e., happy people, erotic) pictures, relative to contamination and attack pictures, led to greater amplitude and velocity of the posterior COP movement during the APAs. The approach-oriented picture categories also increased the displacement of the lateral COP movement in the $\mathrm{S} 1$ region for all participants, as compared with the mutilation and contamination pictures. These results extend prior work finding similar results in healthy young adults (Gelat et al., 2011; Naugle et al., 2011). Thus, the collective data indicate that exposure to approach-oriented stimuli (i.e., pleasant images) facilitates the momentum needed to move forward and reach single limb support during forward gait initiation for persons with PD and healthy older and young adults.

These results have important implication for individuals with PD. Indeed, patients with mild to moderate PD appear capable of planning the spatial and temporal features of the APA prior to initiating gait (Rogers et al., 2011) but exhibit significantly reduced magnitude of the APA (Burleigh Jacobs et al., 1997; Crenna et al., 1990; Halliday et al., 1998). The data from our pre- and posttrials corroborate these studies, showing that persons with PD have similar qualitative patterns of COP movement during gait initiation, 
with reduced COP movement amplitude and velocity particularly during the APAs, ultimately causing a lack of forward momentum production. Thus, induction of pleasant emotional states may be a viable strategy to facilitate the APAs during the initiation of gait in individuals with PD.

No statistically significant emotion modulation was found for the COP movements in the $\mathrm{S} 2$ and $\mathrm{S} 3$ regions of the COP trace. As compared with the APAs (which are more centrally controlled), the S2 and $\mathrm{S} 3$ regions represent the beginning of the locomotor phase of gait initiation, which is generally regulated by lower level spinal processes (Rocchi et al., 2006). Two explanations are offered to account for the diminished impact of emotion on the COP movement in S2 and S3. First, within the present protocol, participants were required to focus more on the planning aspect of the movement (i.e., initiate gait as soon as possible following picture offset), rather than on the locomotor component of the movement (i.e., walk as fast as possible). Consequently, emotion's impact was greatest on $\mathrm{S} 1$ of the COP trajectory and RT, the two measures most reflective of motor-planning processes. Second, the idea that emotion's impact on the preparatory postural adjustments may vary between the APA phase and locomotor phase is supported by animal and human studies. Research has shown that the APAs (S1) are largely controlled by the SMA, premotor cortex, and basal ganglia (Rocchi et al., 2006; Takakusaki et al., 2003), whereas the more automatic locomotor components of gait initiation (i.e., S2, S3) are controlled more so by brainstem and spinal processes (i.e., CPGs; Takakusaki et al., 2003). Thus, the collective processes controlling the APAs are dissimilar to those that control the locomotor phase of the COP movement. As such, the neural circuits regulating the different phases of gait initiation may interact differently with the neural circuits underlying emotional processing.

The results of the present study are in line with evidence suggesting that emotion modulation of movement likely involves higher cortical and subcortical processes. Behavioral work (Coombes et al., 2007) has shown that emotion impacts the speed with which ballistic force is initiated as a function of the impact on centrally driven processes (i.e., cognition, perception), as compared with peripheral processes (i.e., musculature and motor unit recruitment). Furthermore, animal retrograde and anterograde tracing studies (Haber, 2003) and human imaging work (Doron \& Goelman, 2010; Schmidt et al., 2009) suggest that circuits involving the basal ganglia and frontal cortex are critical to the integration of limbic and motor circuits. Nonreciprocal cortico-cortical and corticothalamic pathways may link multiple frontal cortical areas and functional basal ganglia cortical loops, respectively (Haber \& Calzavara, 2009; McFarland \& Haber, 2002; Schmidt et al., 2009). Thus, information can be relayed from the limbic basal ganglia cortical loop to the motor basal ganglia cortical loop allowing emotion to shape motor processes. Moreover, the SN and the subthalamic nucleus (STN) have been identified as potential brain structures that will allow emotion to influence motor processes (Coombes, Corcos, Pavuluri, \& Vaillancourt, in press; Haber, Fudge, \& McFarland, 2000). Specifically, it is thought that the different functional regions of the striatum (i.e., limbic, cognitive, motor) are connected via midbrain dopamine neurons, allowing the ventral limbic region of the striatum to interact with the dorsal motor region. Hence, the collective evidence indicates that the neural circuits underlying the APAs involving cortical and subcortical structures, as compared with the locomotor components of gait initiation, are more likely to be influenced by emotion. Nonetheless, our results suggest that the mechanisms integrating emotion into the motor processes that regulate gait initiation remain intact in PD patients with moderate disease severity while "on" medication.

\section{Emotion's impact on step kinematics}

Our prediction that exposure to the approach-related erotic and happy people pictures would increase the length and velocity of the first step, as compared with all the other picture categories, was partially supported. PD patients showed a greater increase in step velocity of the first step following exposure to the happy people pictures, as compared with the mutilation pictures (which decreased velocity, as compared with neutral pictures). Furthermore, control participants demonstrated a greater increase in step velocity of the first step in response to the erotic, happy people, and mutilation pictures, as compared with the PD patients' response to the mutilation pictures. During the step execution phase of gait initiation, individuals achieve a velocity close to steady state velocity (Brunt et al., 1991). Prior research has shown that the amplitude and velocity of the posterior COP movement during the APAs predict gait velocity at the end of the first step. Thus, the step velocity results, which were relatively small in magnitude, may have been driven by the emotion modulation found in the corresponding APAs. Specifically, the larger APAs found following exposure to the happy people and erotic pictures likely enabled a quicker first step.

While the present data collectively indicate a lesser impact of emotion on the stepping components of gait initiation in PD patients and healthy older adults, it would be inappropriate to conclude that emotion has no impact on steady state walking in this population, since prior work has shown emotional influences on steady walking in young adults (Michalak et al., 2009; Naugle, Joyner, et al., 2010). The effect of emotion on locomotion may depend on the component of gait being evaluated (i.e., planning of the initiation of gait vs. regulation of ongoing gait), the nature of the task instructions (i.e., "walk as soon as possible, but at your normal pace" vs. "walk as quickly as possible"), as well as the temporal 
dynamics of picture presentation and walking (i.e., walking at picture offset vs. walking while viewing picture). Future research should investigate whether emotional state impacts steady state walking in individuals with PD.

Implications and future directions

The findings of the present study provide several potential avenues for future research. First, the results demonstrated that the presentation of pleasant emotional stimuli facilitated gait initiation in PD. The magnitude of the improvements found in the APAs (initial posterior displacement, increase of .33-44 cm for PD patients and .27-33 cm for control), while smaller than those that have been documented with subthalamic deep brain stimulation (DBS) in patients with advanced disease (increase of up to $2 \mathrm{~cm}$; Liu et al., 2006), are similar to improvements found with other acute (stance width; Rocchi et al., 2006) and longitudinal (Tai Chi; Hass et al., 2004) behavioral interventions. Additionally, our results are in line with the phenomenon of paradoxical kinesis - the normalization of movement in PD patients that seems to follow intense externally driven situations such as the sight of fire (Anzak et al., 2011; Daroff, 2008). Indeed, external stimuli with arousing emotional components appear to be able to elicit improvements in motor performance. Thus, further exploration of emotion manipulations as a strategy for optimizing the efficacy of therapeutic interventions in improving gait disturbances may be a promising avenue for future research.

Finally, in the past decade, strides have been made toward greater understanding of the mechanisms allowing emotion to influence motor processes (Coombes et al., in press; Haber, 2003; Schmidt et al., 2009). Nonetheless, the neural system that allows emotional reactivity to modify and guide motor behavior remains poorly understood. Future work could manipulate neurosurgical and pharmacological techniques in persons with PD to further investigate the extent to which basal ganglia function and dopamine are integral to the integration of emotion into motor circuits. Individuals with PD could perform the emotion and movement tasks while "on" and "off' dopaminergic medication and DBS of critical basal ganglia structures, such as the STN and SN. Given that a pathological hallmark of PD is the degeneration of dopaminergic cells, discovering an impaired link between the emotion and motor systems while "off" medication would provide additional confirmation for the importance of functioning dopaminergic pathways in connecting these two circuits in the brain. Additionally, replicating the present findings while "on" DBS of the STN, but not "off" DBS, would indicate that the STN is critical to the integration of emotion information into motor systems controlling gait initiation.

In closing, exposure to threatening stimuli speeded the initiation of the motor response on the gait initiation task, while the approach-related emotional states induced by pleasant pictures clearly facilitated the anticipatory postural adjustments needed to initiate forward gait in healthy older adults and those with PD. The present study provides the first evidence suggesting that the integrity of the circuits integrating the emotion and movement systems in the brain remain largely intact among $\mathrm{PD}$ patients while "on" medication. Thus, manipulating the activation of emotional circuits may be a viable strategy for improving the quality of gait initiation in PD patients. With continued empirical efforts, researchers will be able to inform the development of novel emotion-based interventions that may be integrated with other behavioral, pharmacological, and genetic interventions to optimize motor therapy for medication refractory gait dysfunction in PD.

\section{References}

Anzak, A., Tan, H., Pogosyan, A., Djamshidian, A., Ling, H., Lees, A., \& Brown, P. (2011). Improvements in rate of development and magnitude of force with intense auditory stimuli in patients with Parkinson's disease. European Journal of Neuroscience, 34, 124-132.

Beck, A. T., Steer, R. A., \& Brown, G. K. (1996). Manual for the Beck Depression Inventory-II. San Antonio, TX: Psychological Corporation.

Bowers, D., Miller, K., Mikos, A., Kirsch-Darrow, L., Springer, U., Fernandez, H., \& Okun, E. (2006). Startling facts about emotion in Parkinson's disease: Blunted reactivity to aversive stimuli. Brain, 129, 3356-3365.

Bradley, M. M., Codispoti, M., Sabatinelli, D., \& Lang, P. J. (2001). Emotion and motivation II: Sex differences in picture processing. Emotion, 1, 300-319.

Brunt, D., Lafferty, M. J., McKeon, A., Goode, B., Mulhausen, C., \& Polk, P. (1991). Invariant characteristics of gait initiation. American Journal of Physical Medicine \& Rehabilitation, 70, 206-212.

Burleigh-Jacobs, A., Horak, F. B., Nutt, J. G., \& Obeso, J. A. (1997). Step initiation in Parkinson's disease: influence of levodopa and external sensory triggers. Movement Disorders, 12, 206-215.

Carver, C. S., \& Harmon-Jones, E. (2009). Anger is an approachrelated affect: Evidence and Implications. Psychological Bulletin, 135, 183-204.

Chen, M., \& Bargh, J. A. (1999). Consequences of automatic evaluation: Immediate behavioral predispositions to approach or avoid the stimulus. Personality and Social Psychology Bulletin, $25,215-224$.

Coombes, S. A., Cauraugh, J. H., \& Janelle, C. M. (2007). Emotion and initiating cue alters central and peripheral motor processes. Emotion, 7, 275-284.

Coombes, S. A., Corcos, D. M., Pavuluri, M. N., \& Vaillancourt, D. E. (In press). Maintaining force control despite changes in emotional context engages dorsomedial prefrontal and premotor cortex. Cerebral Cortex

Coombes, S. A., Higgins, T., Gamble, K. M., Cauraugh, J. H., \& Janelle, C. M. (2009). Attentional control theory: Anxiety, emotion, and motor planning. Journal of Anxiety Disorders, 23, 1072-1079.

Crenna, P., Frigo, C., Giovannini, P., \& Piccolo, I. (1990). The initiation of gait in Parkinson's disease. In C. D. Marsden (Ed.), 
Motor disturbances II (pp. 161-173). New York: Academic Press.

Daroff, R. B. (2008). Paradoxical kinesia. Movement Disorders, 23, 1193.

Diermayr, G., Gynsin, P., Hass, C. J., \& Gordon, A. M. (2008). Grip force control during gait initiation with a hand-held object. Experimental Brain Research, 190, 337-345.

Doron, O., \& Goelman, G. (2010). Evidence for asymmetric intra substantia nigra functional connectivity-application to basal ganglia processing. NeuroImage, 49, 2940-2946.

Duckworth, K. L., Bargh, J. A., Garcia, M., \& Chaiken, S. (2002). The automatic evaluation of novel stimuli. Psychological Science, 13, 513-519.

Fahn, S., Elton, R. L., \& UPDRS Development Committee. (1987). Unified Parkinson's Disease Rating Scale. In S. Fahn, C. D. Marsden, D. B. Calne, \& M. Goldstein (Eds.), Recent developments in Parkinson's disease (pp. 153-163). Florham Park, NJ: Macmillan

Frijda, N. H. (2009). Emotion experience and its varieties. Emotion Review, 1, 264-271.

Gélat, T., Coudrat, L., \& Le Pellec, A. (2011). Gait initiation is affected during emotional conflict. Neuroscience Letters, 497, 64-67.

Haber, S. N. (2003). The primate basal ganglia: parallel and integrative networks. Journal of Chemical Neuroanatomy, 26, 317-330.

Haber, S. N., \& Calzavara, R. (2009). The cortico-basal ganglia integrative network: The role of the thalamus. Brain Research Bulletin, 16, 69-74.

Haber, S. N., Fudge, J. L., \& McFarland, N. R. (2000). Striatonigrostriatal pathways in primates form an ascending spiral from the shell to the dorsolateral striatum. Journal of Neuroscience, 20, 2369-2382.

Halliday, G. M., Gai, W. P., Blessing, W. W., \& Geffen, L. B. (1990). Substance P-containing neurons in the pontomesencephalic tegmentum of the human brain. Neuroscience, 39, 81-96.

Halliday, S. E., Winter, D. A., Frank, J. S., Patla, A. E., \& Prince, F. (1998). The initiation of gait in young, elderly, and Parkinson's disease subjects. Gait \& Posture, 8, 8-14.

Harding, A. J., Stimson, E., Henderson, J. M., \& Halliday, G. M. (2002). Clinical correlates of selective pathology in the amygdala of patients with Parkinson disease. Brain, 125, 2431-2445.

Hass, C. J., Gregor, R. J., Waddell, D. E., Oliver, A., Smith, D. W., Fleming, R. P., \& Wolf, S. L. (2004). The influence of Tai Chi training on the center of pressure trajectory during gait initiation in older adults. Archives of Physical Medicine and Rehabilitation, $85,1593-1598$

Hess, U., Blairy, S., \& Kleck, R. E. (2000). The influence of facial emotion displays, gender, and ethnicity on judgments of dominance and affiliation. Journal of Nonverbal Behavior, 24, 265-283.

Jian, Y., Winter, D. A., Ishac, M. C., \& Gilchrist, L. (1993). Trajectory of the body COG and COP during initiation and termination of gait. Gait \& Posture, 1, 9-22.

Lang, P. J. (1980). Behavioral treatment and bio-behavioral assessment: Computer applications. In J. B. Sidowski, J. H. Johnson, \& T. A. Williams (Eds.), Technology in mental health care delivery systems (pp. 119-137). Norwood, NJ: Ablex.

Lang, P. J. (1995). The emotion probe. American Psychologist, 50, $372-385$

Lang, P. J., Bradley, M. M., \& Cuthbert, B. N. (2008). International affective picture system (IAPS): Affective ratings of pictures and instruction manual (Tech. Rep. A-8). Gainesville: University of Florida.

Liu, W., McIntire, K., Kim, S. H., Zhang, J., Dascalos, S., Lyons, K. E., \& Pahwa, R. (2006). Bilateral subthalamic stimulation improves gait initiation in patients with Parkinson's disease. Gait \& Posture, 23, 492-498.

Marsh, A. A., Ambady, N., \& Kleck, R. E. (2005). The effects of fear and anger facial expressions on approach- and avoidance-related behaviors. Emotion, 5, 119-124.

McDonald, W. M., Richard, I. H., \& DeLong, M. (2003). Prevalence, etiology, and treatment of depression in Parkinson's disease. Biological Psychiatry, 54, 363-375.

McFarland, N. R., \& Haber, S. N. (2002). Thalamic relay nuclei of the basal ganglia from both reciprocal and non-reciprocal cortical connections, linking multiple frontal cortical areas. Journal of Neuroscience, 22, 8117-8132.

Michalak, J., Troje, N. F., Fischer, J., Vollmar, P., Heidenreich, T., \& Schulte, D. (2009). Embodiment of sadness and depression-gait patterns associated with dysphoric mood. Psychosomatic Medicine, 71, 580-587.

Miller, K. M., Okun, M. S., Marsiske, M., Fennell, E. B., \& Bowers, D. (2009). Startle reflex hyporeactivity in Parkinson's disease: An emotion-specific or arousal-modulated deficit? Neuropsychologia, 47, 1917-1927.

Morris, M., Iansek, R., Smithson, F., \& Huxham, F. (2000). Postural instability in Parkinson's disease: A comparison with and without a concurrent task. Gait \& Posture, 12, 205-216.

Nasreddine, Z. S., Phillips, N. A., Bédirian, V., Charbonneau, S., Whitehead, V., Collin, I., \& Chertkow, H. (2005). The Montreal Cognitive Assessment, MoCA: A brief screening tool for MCI. Journal of American Geriatric Society, 53, 695-699.

Naugle, K. M., Hass, C. J., Joyner, J., Coombes, S. A., \& Janelle, C. M. (2011). Emotional state affects the initiation of forward gait. Emotion, 11, 267-277.

Naugle, K. M., Joyner, J., Hass, C. J., \& Janelle, C. M. (2010). Emotional influences on locomotor behavior. Journal of Biomechanics, 43, 3099-3103.

Öhman, A., Hamm, A., \& Hugdahl, K. (2000). Cognition and the autonomic nervous system: Orienting, anticipation, and conditioning. In J. Cacioppo, L. Tassinary, \& G. G. Bernston (Eds.), Handbook of psychophysiology (pp. 533-575). New York: Cambridge University Press.

Öhman, A., \& Soares, J. J. (1998). Emotional conditioning to masked stimuli: Expectancies for aversive outcomes following nonrecognized fear-relevant stimuli. Journal of Experimental Psychology. General, 127, 69-82.

Polcyn, A. F., Lipsitz, L. A., Kerrigan, D. C., \& Collins, J. J. (1998). Age-related changes in the initiation of gait: degradation of central mechanisms for momentum generation. Archives of Physical Medicine and Rehabilitation, 79, 1582-1589.

Rocchi, L., Chiari, L., Cappello, A., \& Horak, F. B. (2006). Identification of distinct characteristics of postural sway in Parkinson's disease: A feature selection procedure based on principal component analysis. Neuroscience Letters, 394, 140 145.

Rodriguez-Oroz, M. C., Obeso, J. A., Lang, A. E., Houeto, J. L., Pollak, P., \& Rehncrona, S. (2005). Bilateral deep brain stimulation in Parkinson's disease: A multicenter study with 4 years follow-up. Brain, 128, 2240-2249.

Rogers, M. W., Kennedy, R., Palmer, S., Pawar, M., Reising, M., Martinez, K. M., \& MacKinnon, C. D. (2011). Postural preparation prior to stepping in patients with Parkinson's disease. Journal of Neurophysiology, 106, 915-928.

Schmidt, L., Clery-Melin, M., Lafargu, G., Valabregue, R., Fossati, P., Dubois, B., \& Pessiglione, M. (2009). Get aroused and be stronger: Emotional facilitation of physical effort in the human brain. Journal of Neuroscience, 29, 9450-9457.

Sethi, K. (2008). Levodopa unresponsive symptoms in Parkinson disease. Movement Disorders, 23(Suppl. 3), S521-S533. 
Slaughter, J. R., Slaughter, K. A., Nichols, D., Holmes, S. E., \& Martens, M. P. (2001). Prevalence, clinical manifestations, etiology, and treatment of depression in Parkinson disease. Journal of Neuropsychiatry and Clinical Neuroscience, 13, 187-196.

Spielberger, C. D. (1983). Manual for the State-Trait Anxiety Inventory (STAI). Palto Alto, CA: Consulting Psychologists Press.

Starkstein, S. E., Migliorelli, R., Manes, F., Teson, A., Petracca, G., Chemerinski, E., \& Leiguarda, R. (1995). The prevalence and clinical correlates of apathy and irritability in Alzheimer's disease. European Journal of Neurology, 2, 540-546.

Stins, J. F., \& Beek, P. J. (2011). Organization of voluntary stepping in response to emotion-inducing pictures. Gait \& Posture, 34, 164-168.

Takakusaki, K., Habaguchi, T., Ohtinata-Sugimoto, J., Saitoh, K., \& Sakamoto, T. (2003). Basal ganglia efferents to the brainstem centers controlling postural muscle tone and locomotion: A new concept for understanding motor disorders in basal ganglia dysfunction. Neuroscience, 119, 293-308.

Uhl, G. R., Hedreen, J. C., \& Price, D. L. (1985). Parkinson's disease: Loss of neurons from the ventral tegmental area contralateral to therapeutic surgical lesions. Neurology, 35, 1215-1218.

Watson, D., Clarke, L. A., \& Tellegen, A. (1988). Developmental and validation of brief measures of positive and negative affect: The PANAS scales. Journal of Personality and Social Psychology, 54, 1063-1070.

Zettel, J. L., McIlroy, W. E., \& Maki, B. E. (2002). Environmental constraints on foot trajectory reveal the capacity for modulation of anticipatory postural adjustments during rapid triggered stepping reactions. Experimental Brain Research, 146, 38-47. 